\title{
Cell-Type-Specific Metabolic Profiling Achieved by Combining De- sorption Electrospray Ionization Mass Spectrometry Imaging and Immunofluorescence Staining
}

Xin Yan, ${ }^{\ddagger, a b}$ Xiaoai Zhao, ${ }^{\ddagger, c}$ Zhenpeng Zhou, ${ }^{\ddagger}, \mathrm{a}$ Andrew McKay, ${ }^{c}$ Anne Brunet, ${ }^{*}, \mathrm{~cd}$ and Richard N. Zare, ${ }^{*}$ a

aDepartment of Chemistry, Stanford University, Stanford, CA 94305, USA. E-mail: rnz@stanford.edu

bDepartment of Chemistry, Texas A\&M University, College Station, TX 77843.

'Department of Genetics, Stanford University, Stanford, CA 94305

dGlenn Laboratories for the Biology of Aging, Stanford University, Stanford, CA 94305, USA.

E-mail: anne.brunet@stanford.edu

Electronic Supplementary Information (ESI) available: MS data and mouse brain images. See DOI: 10.1039/x0xx00000x

$\ddagger$ These authors contributed equally. 
S1. Laboratory animals. Ten mice used in this study were 4-month-old male C57BL/6 mice from the NIA Aged Rodent colony. Mice were habituated for more than one week at Stanford before use. At Stanford, all mice were housed in the Comparative Medicine Pavilion, and their care was monitored by the Veterinary Service Center at Stanford University under IUCAC protocols 8661.

S2. Sample preparation. Mouse brains were dissected, and immediately snap frozen on dry ice. Brains were cut into sections at $-20^{\circ} \mathrm{C}$ on a Leica CM3050S cryostat. Brains were not embedded in optimal cutting temperature (OCT) polymeric compound to avoid background compound peaks from residual OCT during tissue sectioning. Instead, a minimal amount of OCT was applied to attach the cerebellum of the brain tissue to the mounting plate before sectioning. Coronal sections of $16 \mu \mathrm{m}$ thickness were generated between Bregma 0.3-0.8 $\mathrm{mm}$ in the mouse forebrain, thawmounted onto slides and stored at $-80{ }^{\circ} \mathrm{C}$ without fixation. Tissue slides were dried for 20 min at room temperature using a vacuum desiccator before DESI-MSI experiments.

\section{S3. Co-registration of MSI images and fluorescence images}

MSI images with pixel compositions were then overlaid with fluorescence images by inputting both images to the imaging software MSireader, aligning two images in a single coordinated system manually using the image overlay tool. Anatomical landmarks such as corpus callosum were used to facilitate fast overlay. Pixel information of metabolic distribution, cell numbers, and cell types were then extracted from each MSI pixel. Registration error was evaluated by comparing cell number and composition in one MS pixel between the overlay images of the same fluorescence images and DESI-MS images performed at different times. The differences in cell number and composition are less than $1 \%$.

\section{S4. Metabolite Identification}

Structures of molecular species were identified using high mass accuracy measurements and tandem mass spectrometry analysis (collision induced dissociation (CID)). The ions of metabolites in the MS data were searched in the LipidMaps database (http://www.lipidmaps.org/) and Metlin database (https://metlin.scripps.edu) based on high mass accuracy and their isotopic distribution. When the database listed multiple isobaric/isomeric species, CID was performed and compared with that of the standard from the above database and reported literature to characterize the species. Representative tandem mass spectra were shown in Figure S4. For the ions of which the abundance was not high enough to fulfill tandem mass spectrometry, only high mass accuracy measurement was utilized, and the molecular formula with lowest mass error was assigned to corresponding $\mathrm{m} / \mathrm{z}$ values (mass tolerance within 0.005 ). Lipids that are commercially available (e.g. oleic acid, PG(36:2), and PS(36:2)) were identified by comparing their tandem MS data with standards.

\section{S5. Cosine similarity tests}

We performed cosine similarity tests between deconvoluted mass spectra of neurons and astrocytes of each individual animal, and spectra from all 10 pixels of the same animal. We then assessed correlation between cell type composition of the two cell types - astrocyte or neuron on each pixel with cosine similarity score obtained from comparing to respective cell type-specific spectra of the same animal. We observed a strong positive correlation across almost all samples for both neurons and astrocytes (Figure S6 and S7). This suggests that pixels that have a higher percentage of a given cell type better represent the deconvoluted spectra of the same cell type than pixels that have smaller presence of the same cell type. In addition, the majority of observed positive correlation is significant with $p$-value $<0.05$. 

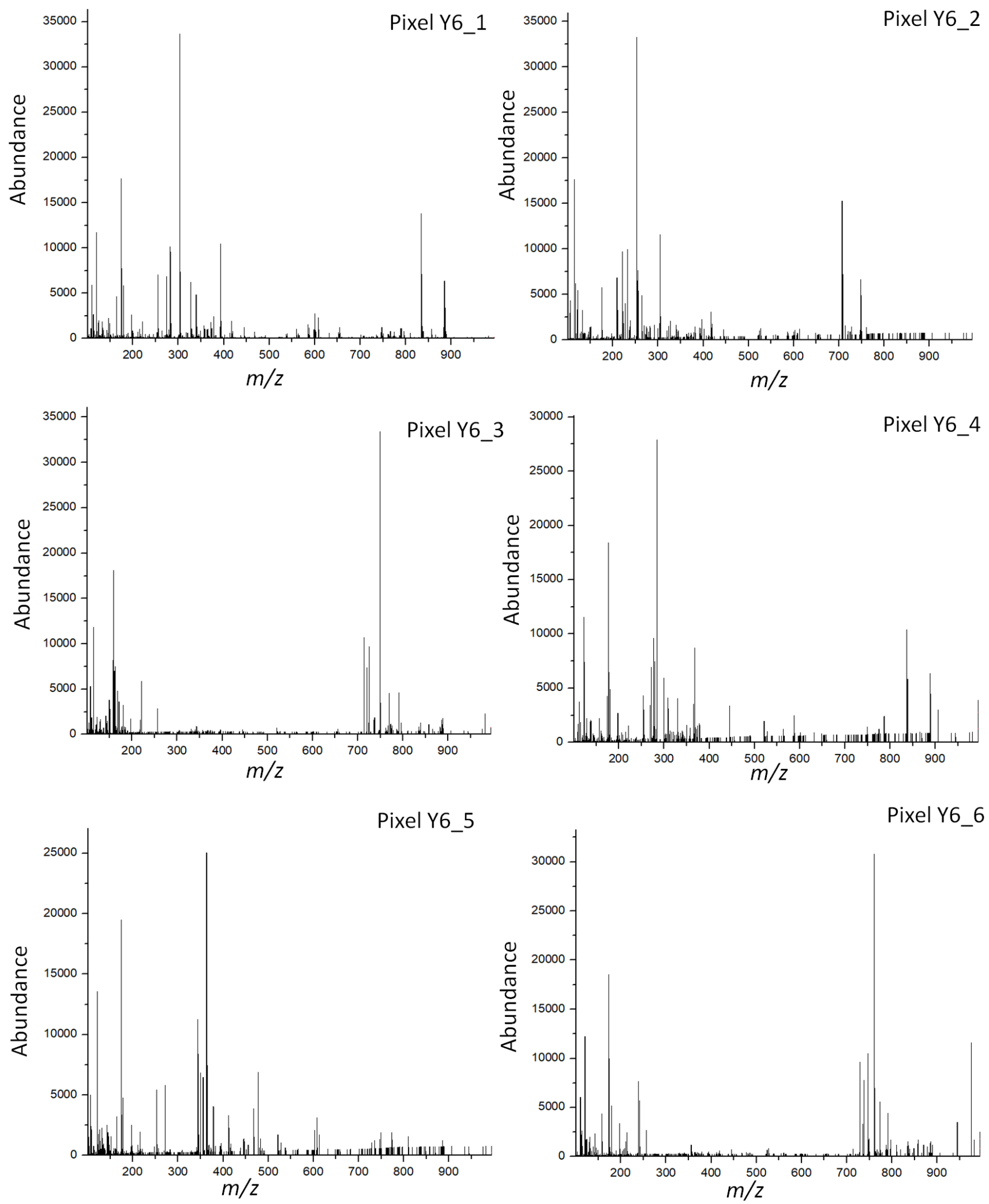

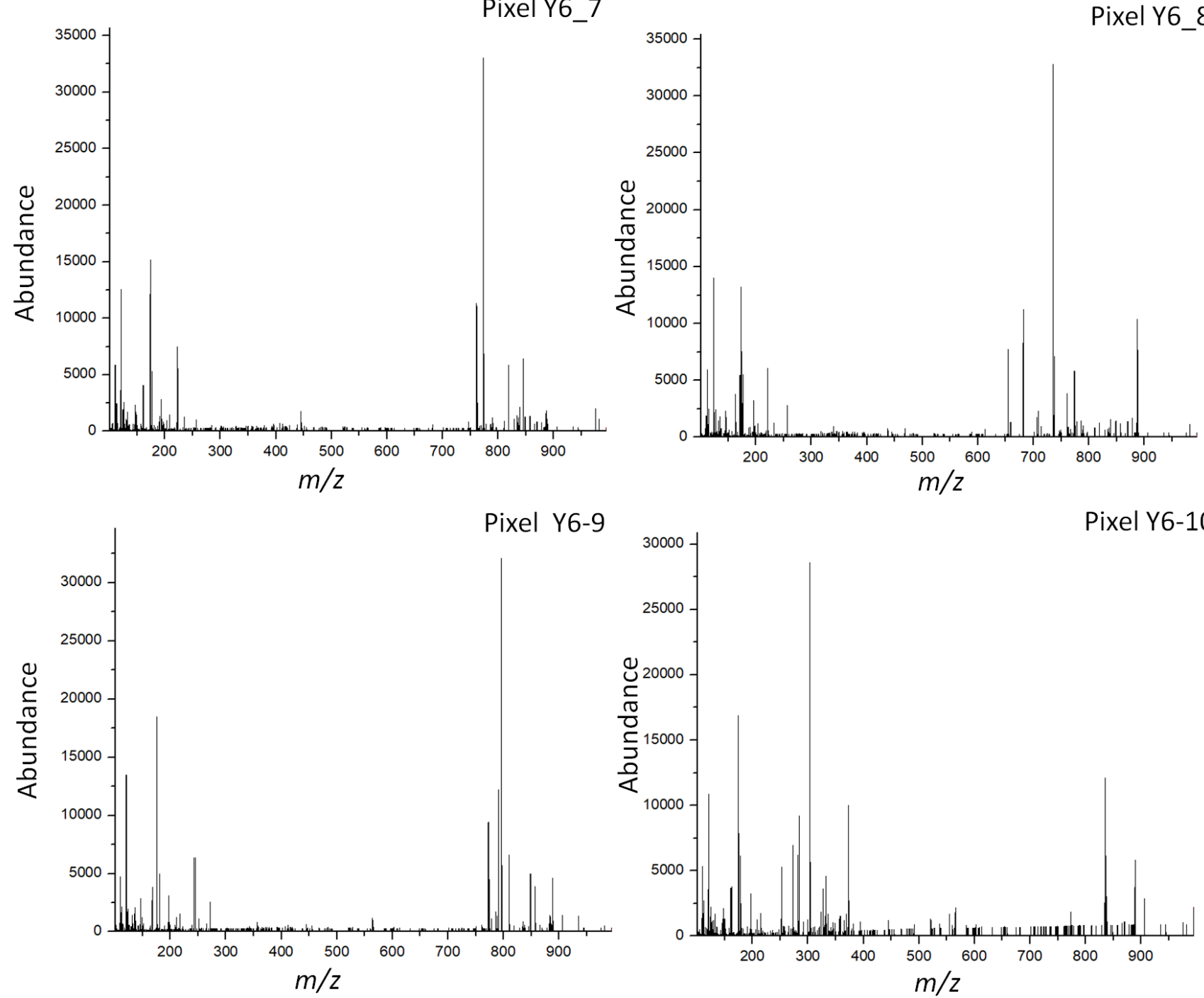

Figure S1. Representative metabolic profiles of pixels that include variety of cell types and numbers from mouse brain sections (see Table S2 for cell compositions of pixels). 
a) Mouse Brain Reference Atlas

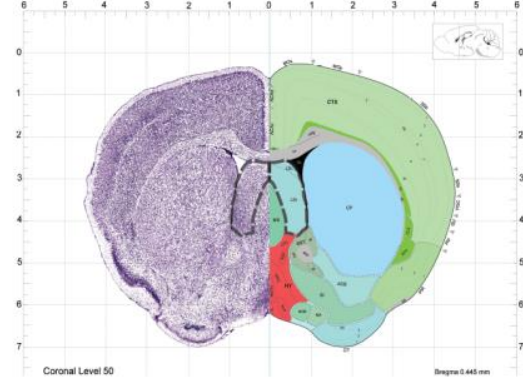

d) Myelin Basic Protein (Mbp) -
Oligodendrocyte Marker

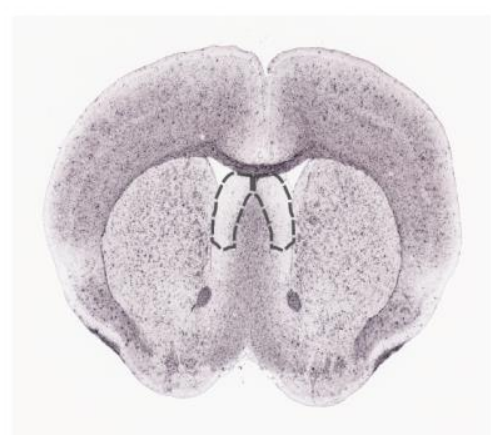

b) Microtubule Associated Protein 2 (Map2) - Neuron Marker

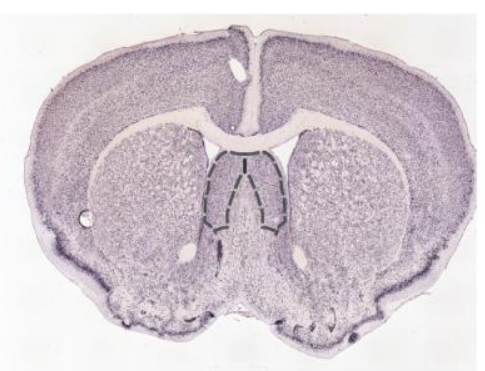

e) Allograft Inflammatory Factor a (Aif1, as known as Iba1) - Microglia Marker

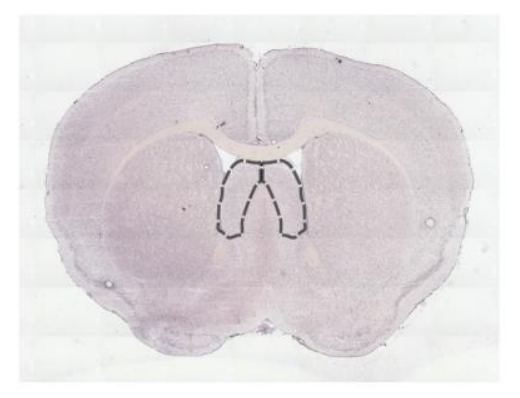

c) Glial Fibrillary Acidic Protein (Gfap) Astrocyte Marker

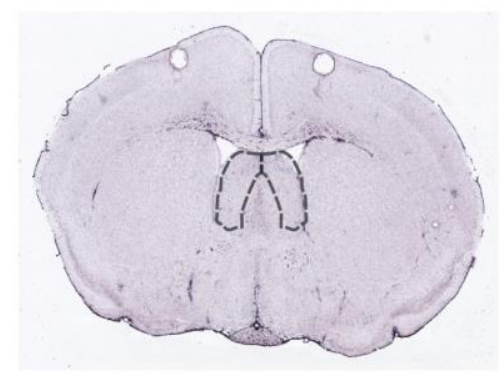

Figure S2. Cell-type-specific marker gene visualized by in situ hybridization in the coronal section of mouse forebrain. All images are coronal section (Bregma 0.4-0.6mm) obtained from Allen Developing Mouse Brain Atlas. ${ }^{54-55}$ a) Lateral septal nucleus in both hemisphere (areas circled with black dashed line) is traced on mouse brain reference atlas. Gene-specific localization is shown in b) neuron marker Map2; c) astrocyte marker Gfap; d) oligodendrocyte marker Mbp; and e) microglia marker Aif1. Dark brown indicates positive detection of mRNA. Robust levels of neuron (b) and astrocyte (c) specific marker gene are detected in the lateral septal nucleus region, whereas oligodendrocyte (d) and microglia (e) specific marker genes display low to undetectable level in this region of the brain. 


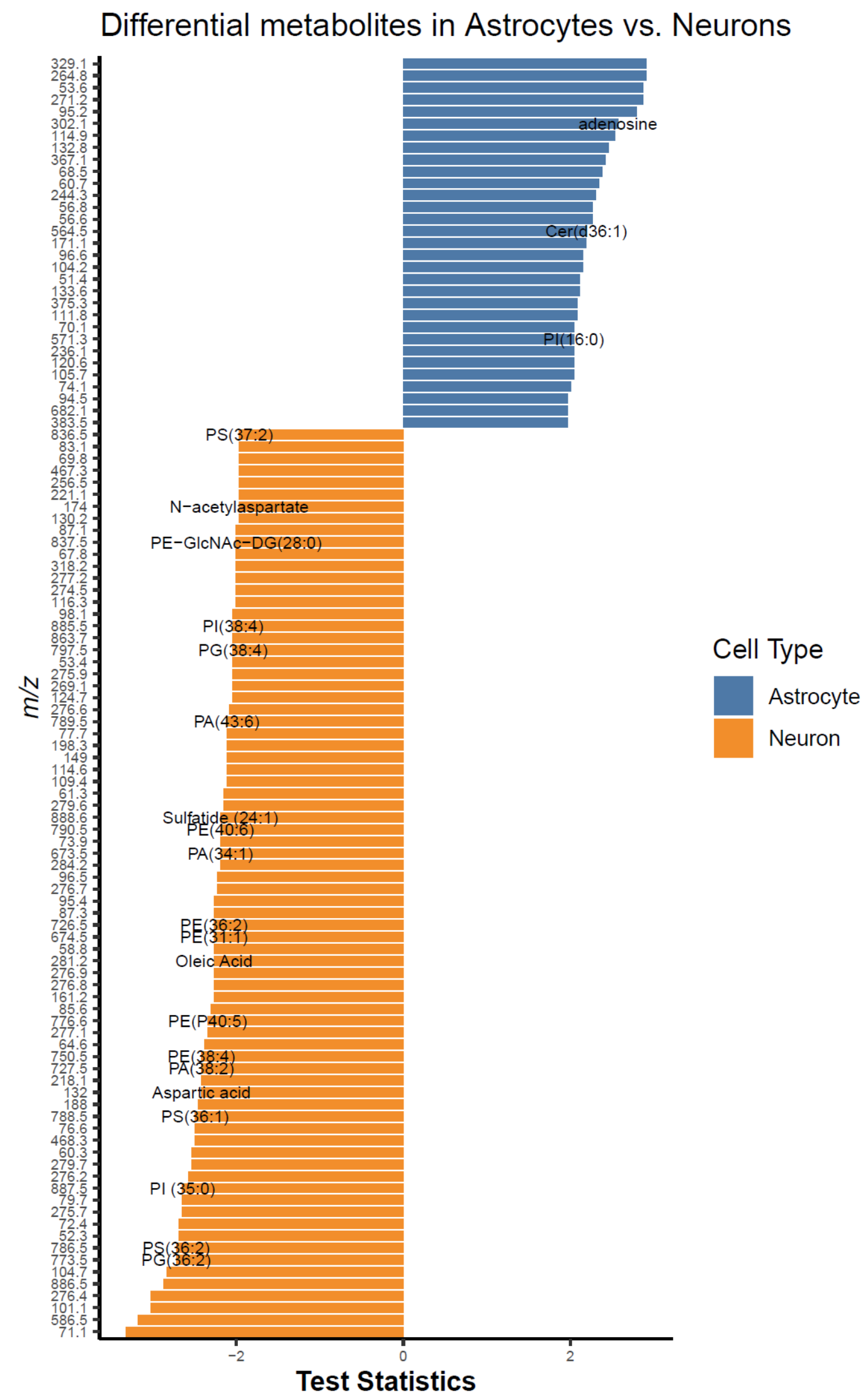

Figure S3. Wilcoxon's rank-sum test result showing the $\mathrm{m} / \mathrm{z}$ of 31 metabolites displaying enriched intensities in astrocytes and 76 metabolites showing accumulation in neurons. 

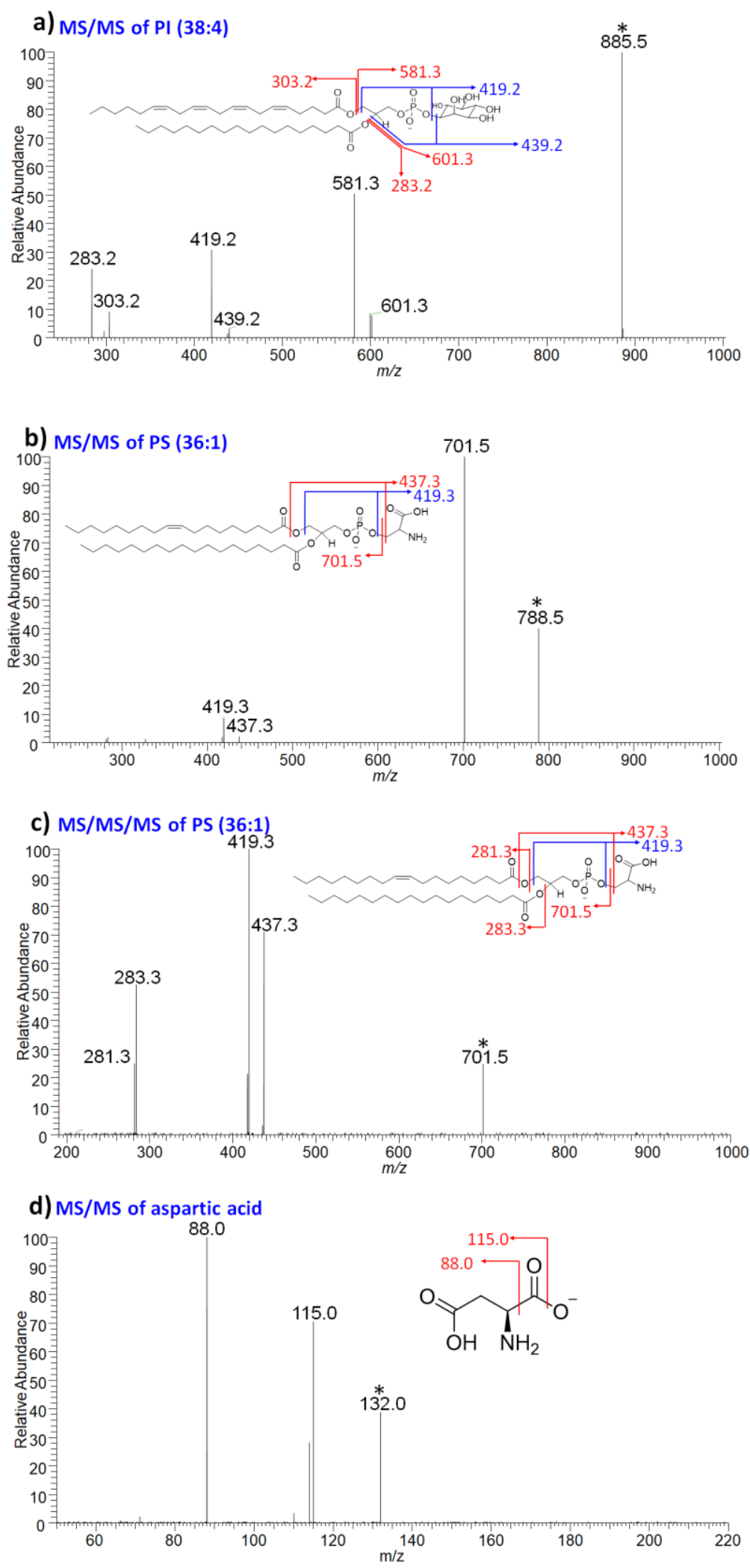

Figure S4. Tandem MS of representative metabolites. a) MS/MS spectrum of ions at $m / z 885.5 ; b$ ) MS/MS spectrum of ions at $\mathrm{m} / \mathrm{z} 788.5$; c) MS/MS/MS spectrum of ions at $\mathrm{m} / \mathrm{z} 788.5$; and d) MS/MS spectrum of ions at $m / z$ 132.0. ${ }^{*}$ represents the parent ions in tandem MS. 


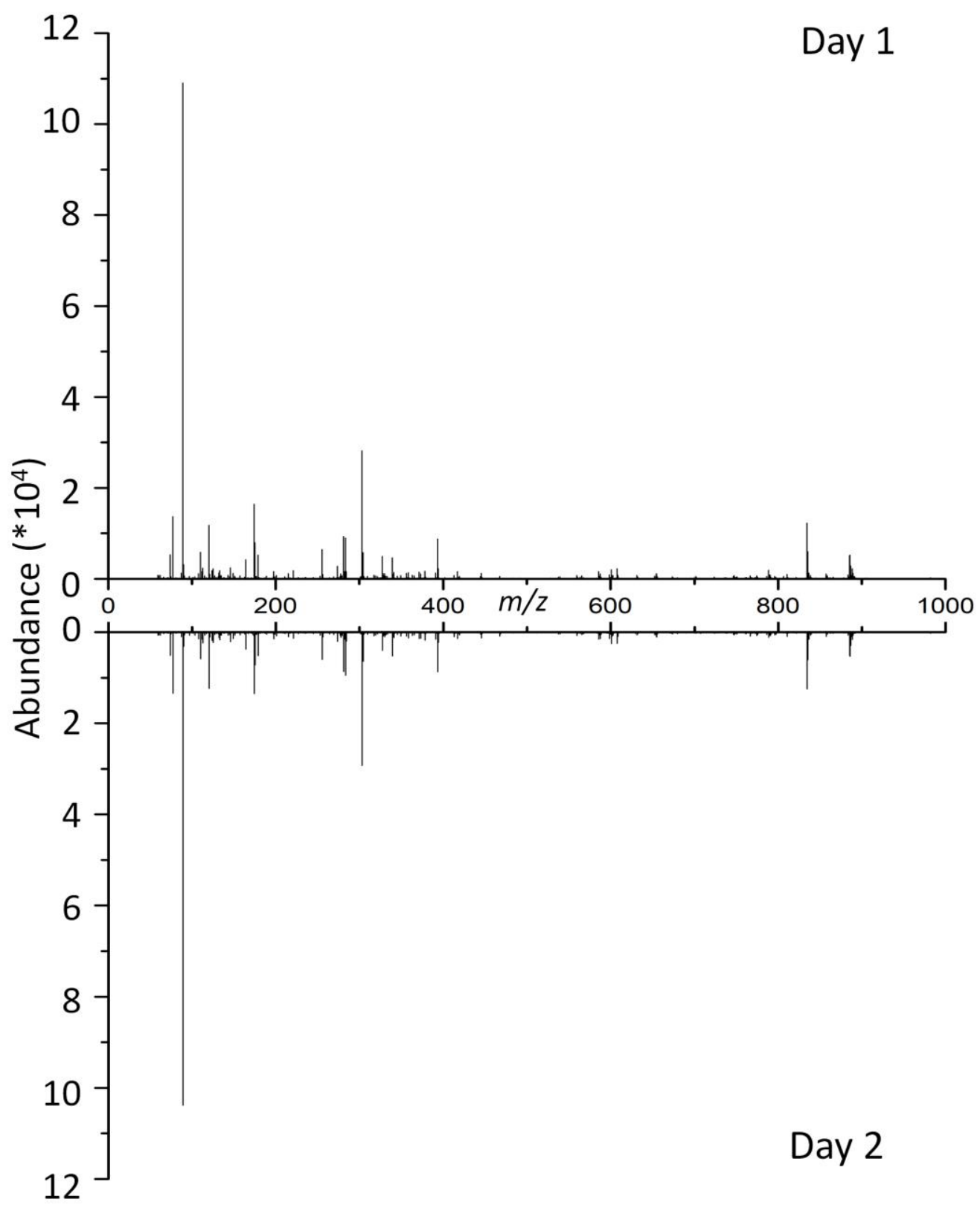

Figure S5. Representative mass spectra of a pixel obtained from DESI-MSI of the same tissue section on different days. 

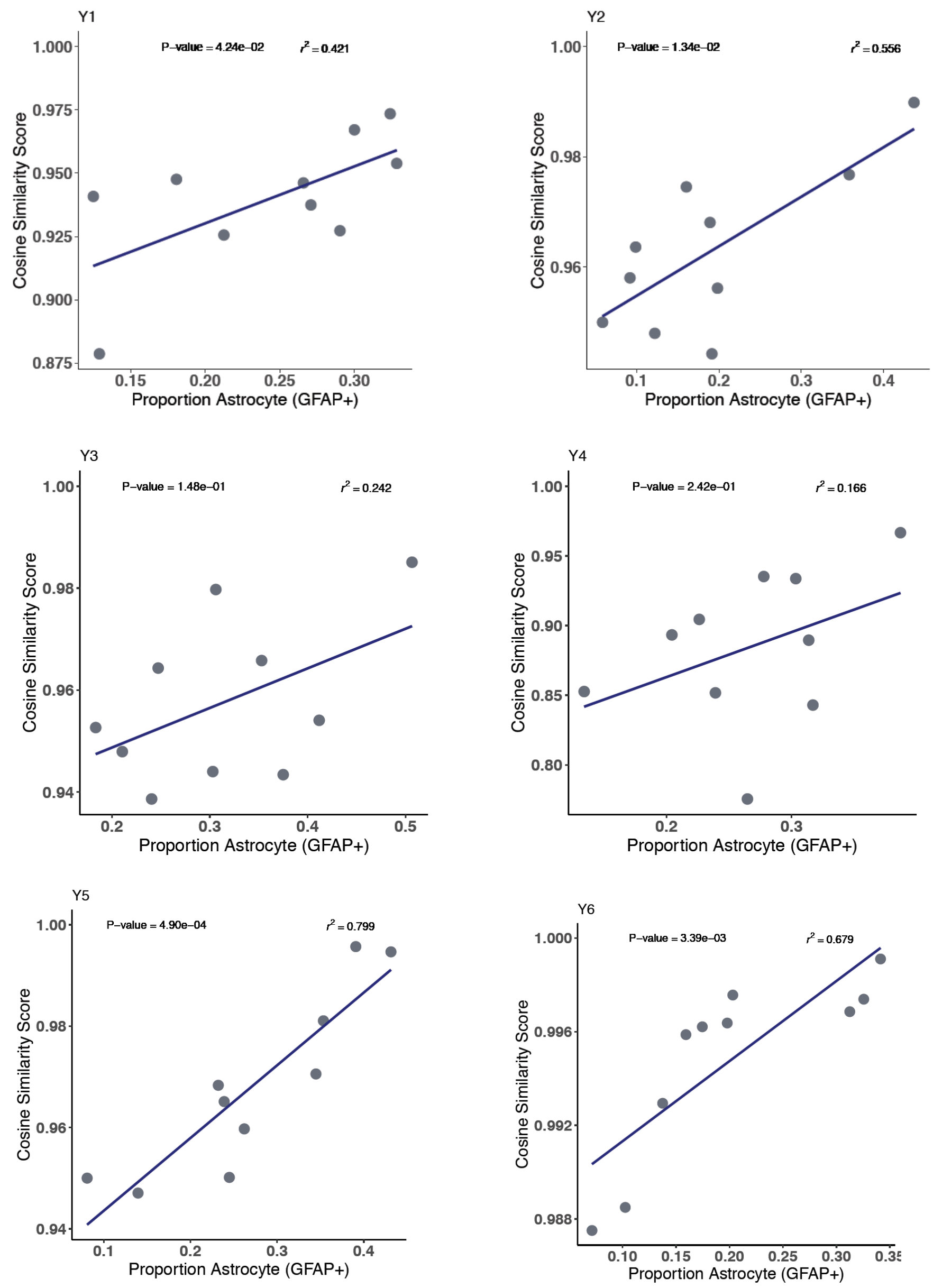

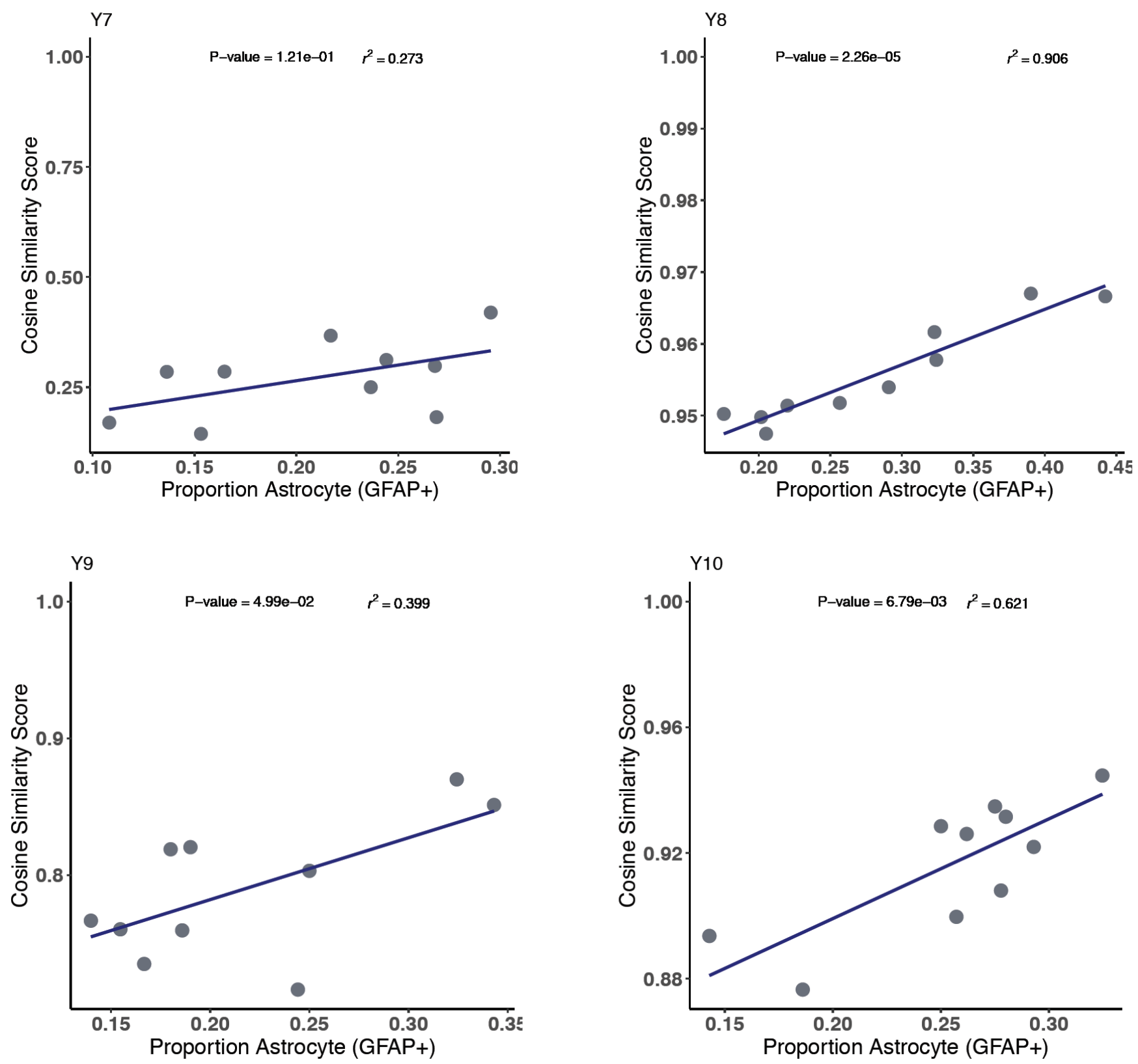

Figure S6. Cosine similarity test of astrocytes (GFAP Positive). Cosine similarity tests were done between deconvoluted mass spectra of astrocytes of each individual animal, and spectra from all 10 pixels of the same animal. Correlation analysis was performed between cell type composition of astrocyte on each pixel with cosine similarity score obtained from comparing to deconvoluted astrocytes-specific spectra of the same animal. 

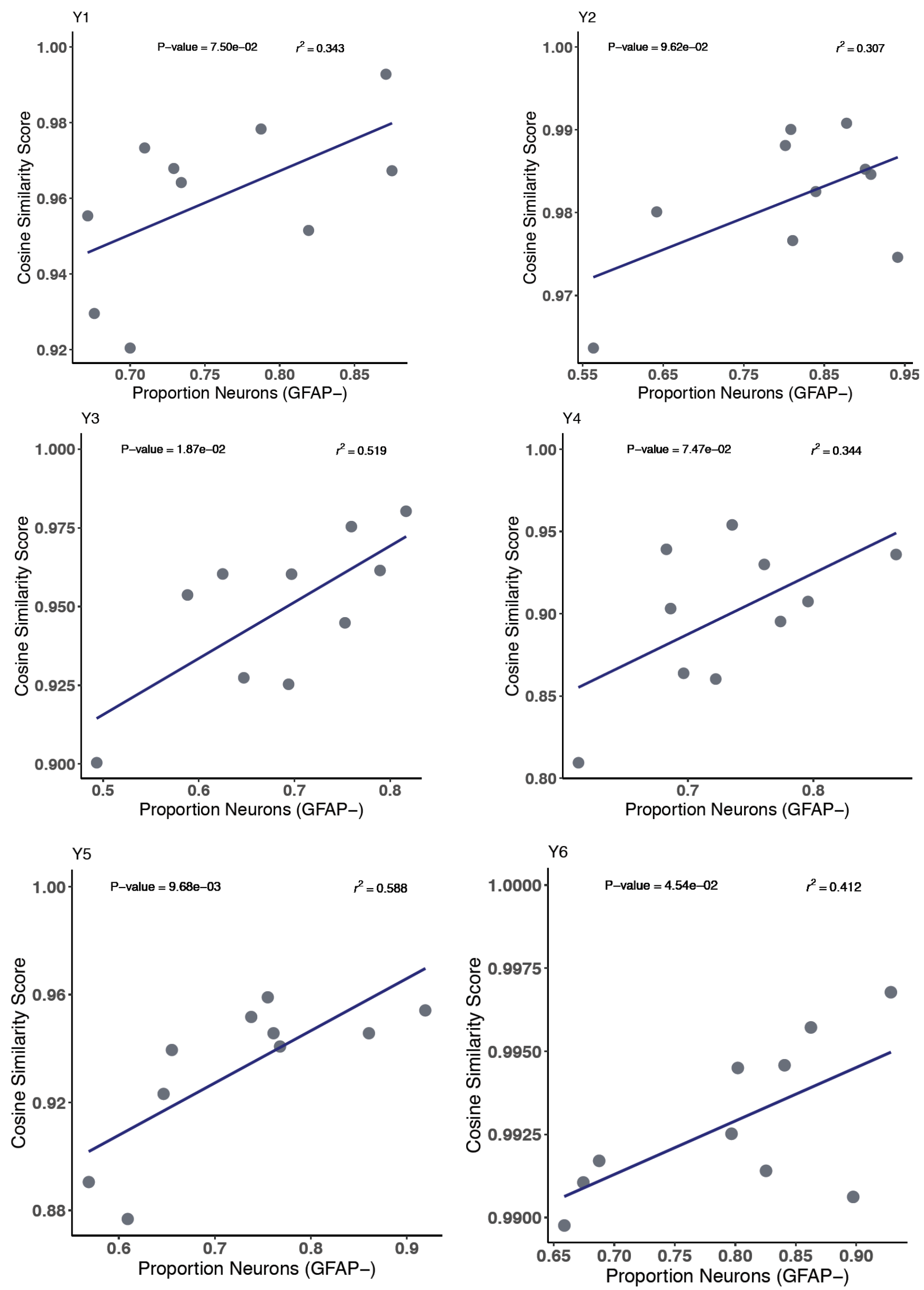

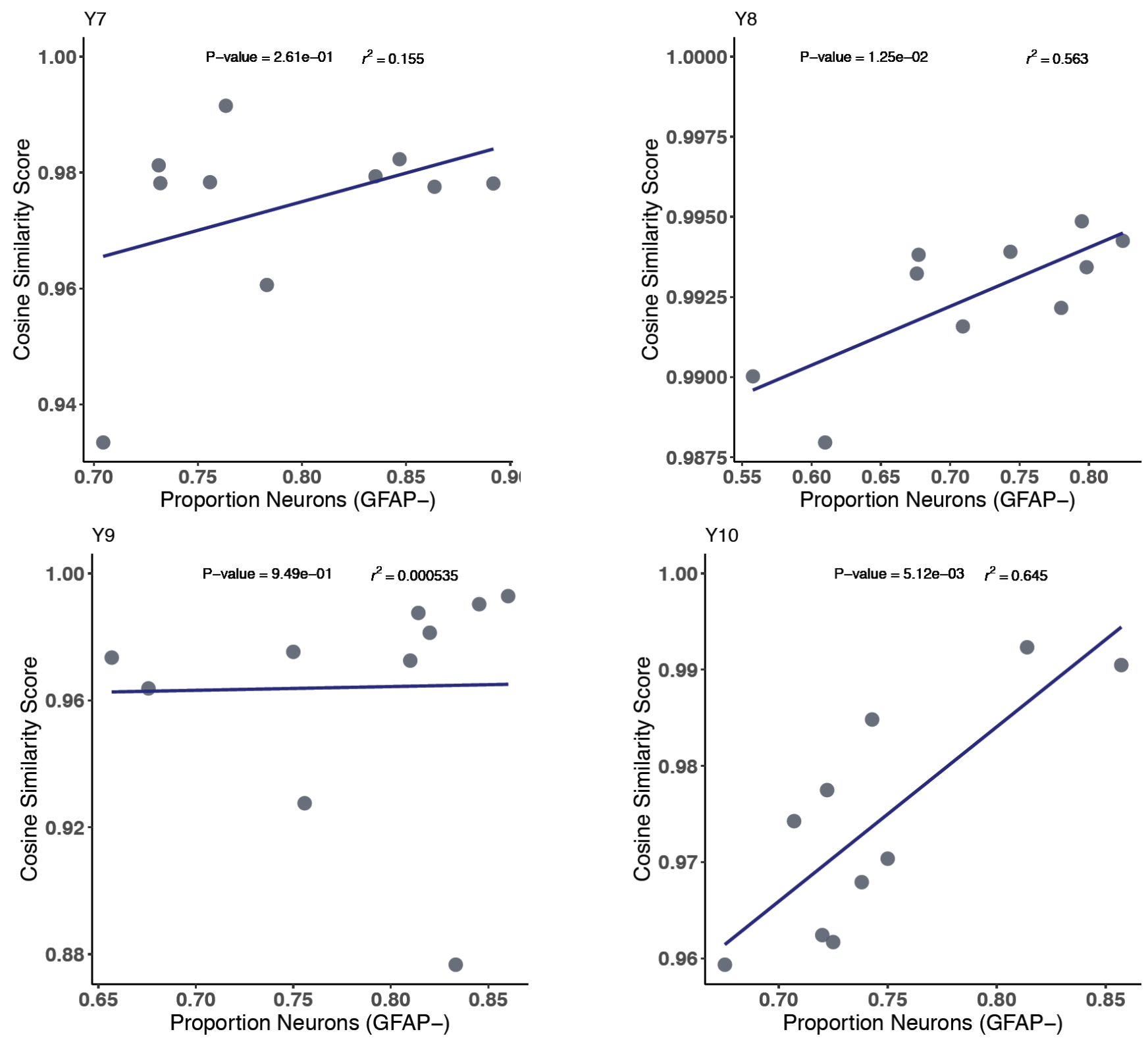

Figure S7. Cosine similarity test of neurons (GFAP Negative). Cosine similarity tests were done between deconvoluted mass spectra of neurons of each individual animal, and spectra from all 10 pixels of the same animal. Correlation analysis was performed between cell type composition of neurons on each pixel with cosine similarity score obtained from comparing to deconvoluted neurons-specific spectra of the same animal. 
Table S1: Cell compositions of DESI-MSI pixels (100 pixels total)

\begin{tabular}{|c|c|c|c|c|c|}
\hline Pixel & $\begin{array}{l}\text { Percentage } \\
\text { of astrocytes }\end{array}$ & $\begin{array}{l}\text { Percentage } \\
\text { of neurons }\end{array}$ & $\begin{array}{l}\text { Total cell } \\
\text { count }\end{array}$ & $\begin{array}{l}\text { Cell count of as- } \\
\text { trocytes }\end{array}$ & $\begin{array}{l}\text { Cell count of neu- } \\
\text { rons }\end{array}$ \\
\hline $\begin{array}{l}\text { Y1_1 } \\
\end{array}$ & 0.270833 & 0.729167 & 48 & 13 & 35 \\
\hline Y1_2 & 0.328358 & 0.671642 & 67 & 22 & 45 \\
\hline Y1_3 & 0.180723 & 0.819277 & 83 & 15 & 68 \\
\hline $\begin{array}{l}\text { Y1_4 } \\
\end{array}$ & 0.125 & 0.875 & 72 & 9 & 63 \\
\hline Y1_5 & 0.129032 & 0.870968 & 93 & 12 & 81 \\
\hline $\begin{array}{l}\text { Y1_6 } \\
\text { 1 }\end{array}$ & 0.3 & 0.7 & 60 & 18 & 42 \\
\hline $\begin{array}{l}\text { Y1_7 } \\
\text { 1 }\end{array}$ & 0.323944 & 0.676056 & 71 & 23 & 48 \\
\hline 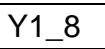 & 0.290323 & 0.709677 & 62 & 18 & 44 \\
\hline Y1_9 & 0.2125 & 0.7875 & 80 & 17 & 63 \\
\hline Y1_10 & 0.265823 & 0.734177 & 79 & 21 & 58 \\
\hline Y2_1 & 0.09901 & 0.90099 & 101 & 10 & 91 \\
\hline Y2_2 & 0.122222 & 0.877778 & 90 & 11 & 79 \\
\hline Y2_3 & 0.092105 & 0.907895 & 76 & 7 & 69 \\
\hline Y2_4 & 0.191489 & 0.808511 & 94 & 18 & 76 \\
\hline Y2_5 & 0.198276 & 0.801724 & 116 & 23 & 93 \\
\hline Y2_6 & 0.160494 & 0.839506 & 81 & 13 & 68 \\
\hline Y2_7 & 0.058824 & 0.941176 & 85 & 5 & 80 \\
\hline Y2_8 & 0.358025 & 0.641975 & 81 & 29 & 52 \\
\hline Y2_9 & 0.43662 & 0.56338 & 71 & 31 & 40 \\
\hline Y2_10 & 0.189189 & 0.810811 & 74 & 14 & 60 \\
\hline Y3_1 & 0.375 & 0.625 & 80 & 30 & 50 \\
\hline Y3_2 & 0.411765 & 0.588235 & 85 & 35 & 50 \\
\hline Y3_3 & 0.247191 & 0.752809 & 89 & 22 & 67 \\
\hline Y3_4 & 0.210526 & 0.789474 & 57 & 12 & 45 \\
\hline Y3_5 & 0.506667 & 0.493333 & 75 & 38 & 37 \\
\hline Y3_6 & 0.352941 & 0.647059 & 102 & 36 & 66 \\
\hline Y3_7 & 0.306122 & 0.693878 & 98 & 30 & 68 \\
\hline Y3_8 & 0.240506 & 0.759494 & 79 & 19 & 60 \\
\hline Y3_9 & 0.183333 & 0.816667 & 120 & 22 & 98 \\
\hline Y3_10 & 0.30303 & 0.69697 & 66 & 20 & 46 \\
\hline Y4_1 & 0.22619 & 0.77381 & 84 & 19 & 65 \\
\hline Y4_2 & 0.313725 & 0.686275 & 102 & 32 & 70 \\
\hline Y4_3 & 0.277778 & 0.722222 & 108 & 30 & 78 \\
\hline Y4_4 & 0.303371 & 0.696629 & 89 & 27 & 62 \\
\hline Y4_5 & 0.264706 & 0.735294 & 102 & 27 & 75 \\
\hline Y4_6 & 0.387097 & 0.612903 & 93 & 36 & 57 \\
\hline Y4_7 & 0.317073 & 0.682927 & 82 & 26 & 56 \\
\hline Y4_8 & 0.134146 & 0.865854 & 82 & 11 & 71 \\
\hline Y4_9 & 0.204301 & 0.795699 & 93 & 19 & 74 \\
\hline Y4_10 & 0.23913 & 0.76087 & 92 & 22 & 70 \\
\hline Y5_1 & 0.244898 & 0.755102 & 98 & 24 & 74 \\
\hline Y5_2 & 0.353448 & 0.646552 & 116 & 41 & 75 \\
\hline Y5_3 & 0.431373 & 0.568627 & 102 & 44 & 58 \\
\hline Y5_4 & 0.390805 & 0.609195 & 87 & 34 & 53 \\
\hline Y5_5 & 0.344828 & 0.655172 & 87 & 30 & 57 \\
\hline Y5_6 & 0.232143 & 0.767857 & 112 & 26 & 86 \\
\hline Y5_7 & 0.238938 & 0.761062 & 113 & 27 & 86 \\
\hline Y5_8 & 0.080808 & 0.919192 & 99 & 8 & 91 \\
\hline Y5_9 & 0.139535 & 0.860465 & 86 & 12 & 74 \\
\hline
\end{tabular}




\begin{tabular}{|c|c|c|c|c|c|}
\hline Y5_10 & 0.262136 & 0.737864 & 103 & 27 & 76 \\
\hline Y6_1 & 0.203125 & 0.796875 & 128 & 26 & 102 \\
\hline Y6_2 & 0.197917 & 0.802083 & 96 & 19 & 77 \\
\hline Y6_3 & 0.071429 & 0.928571 & 84 & 6 & 78 \\
\hline Y6_4 & 0.1375 & 0.8625 & 80 & 11 & 69 \\
\hline Y6_5 & 0.102564 & 0.897436 & 78 & 8 & 70 \\
\hline Y6_6 & 0.34127 & 0.65873 & 126 & 43 & 83 \\
\hline Y6_7 & 0.3125 & 0.6875 & 96 & 30 & 66 \\
\hline Y6_8 & 0.174603 & 0.825397 & 63 & 11 & 52 \\
\hline Y6_9 & 0.159292 & 0.840708 & 113 & 18 & 95 \\
\hline Y6_10 & 0.325581 & 0.674419 & 86 & 28 & 58 \\
\hline Y7_1 & 0.295455 & 0.704545 & 88 & 26 & 62 \\
\hline Y7_2 & 0.216867 & 0.783133 & 83 & 18 & 65 \\
\hline Y7_3 & 0.244186 & 0.755814 & 86 & 21 & 65 \\
\hline Y7_4 & 0.268041 & 0.731959 & 97 & 26 & 71 \\
\hline Y7_5 & 0.268817 & 0.731183 & 93 & 25 & 68 \\
\hline Y7_6 & 0.153153 & 0.846847 & 111 & 17 & 94 \\
\hline Y7_7 & 0.108108 & 0.891892 & 74 & 8 & 66 \\
\hline Y7_8 & 0.236559 & 0.763441 & 93 & 22 & 71 \\
\hline Y7_9 & 0.136364 & 0.863636 & 88 & 12 & 76 \\
\hline Y7_10 & 0.164706 & 0.835294 & 85 & 14 & 71 \\
\hline Y8_1 & 0.175573 & 0.824427 & 131 & 23 & 108 \\
\hline Y8_2 & 0.442308 & 0.557692 & 156 & 69 & 87 \\
\hline Y8_3 & 0.390244 & 0.609756 & 123 & 48 & 75 \\
\hline Y8_4 & 0.256637 & 0.743363 & 113 & 29 & 84 \\
\hline Y8_5 & 0.290909 & 0.709091 & 110 & 32 & 78 \\
\hline Y8_6 & 0.324138 & 0.675862 & 145 & 47 & 98 \\
\hline Y8_7 & 0.322835 & 0.677165 & 127 & 41 & 86 \\
\hline Y8_8 & 0.205128 & 0.794872 & 117 & 24 & 93 \\
\hline Y8_9 & 0.201681 & 0.798319 & 119 & 24 & 95 \\
\hline Y8_10 & 0.22 & 0.78 & 100 & 22 & 78 \\
\hline Y9_1 & 0.324324 & 0.675676 & 111 & 36 & 75 \\
\hline Y9_2 & 0.343137 & 0.656863 & 102 & 35 & 67 \\
\hline Y9_3 & 0.244186 & 0.755814 & 86 & 21 & 65 \\
\hline Y9_4 & 0.166667 & 0.833333 & 84 & 14 & 70 \\
\hline Y9_5 & 0.185841 & 0.814159 & 113 & 21 & 92 \\
\hline Y9_6 & 0.19 & 0.81 & 100 & 19 & 81 \\
\hline Y9_7 & 0.139785 & 0.860215 & 93 & 13 & 80 \\
\hline Y9_8 & 0.18 & 0.82 & 100 & 18 & 82 \\
\hline Y9_9 & 0.154639 & 0.845361 & 97 & 15 & 82 \\
\hline Y9_10 & 0.25 & 0.75 & 88 & 22 & 66 \\
\hline Y10_1 & 0.261905 & 0.738095 & 84 & 22 & 62 \\
\hline Y10_2 & 0.277778 & 0.722222 & 108 & 30 & 78 \\
\hline Y10_3 & 0.28 & 0.72 & 75 & 21 & 54 \\
\hline Y10_4 & 0.275 & 0.725 & 80 & 22 & 58 \\
\hline Y10_5 & 0.324675 & 0.675325 & 77 & 25 & 52 \\
\hline Y10_6 & 0.25 & 0.75 & 76 & 19 & 57 \\
\hline Y10_7 & 0.257143 & 0.742857 & 105 & 27 & 78 \\
\hline Y10_8 & 0.292929 & 0.707071 & 99 & 29 & 70 \\
\hline Y10_9 & 0.142857 & 0.857143 & 98 & 14 & 84 \\
\hline Y10_10 & 0.186047 & 0.813953 & 86 & 16 & 70 \\
\hline
\end{tabular}


Table S2: Identified Metabolites in Murine Neurons and Astrocytes using Cell-Type-Specific DESI-MSI

\begin{tabular}{|c|c|c|c|c|c|c|}
\hline $\begin{array}{c}\text { Observed } \\
\mathrm{m} / \mathrm{z}\end{array}$ & Formula & $\begin{array}{c}\text { Theoretical } \\
\mathrm{m} / \mathrm{z}\end{array}$ & $\begin{array}{c}\text { Mass } \\
\text { Difference }\end{array}$ & $\mathrm{ppm}$ & $\begin{array}{l}\text { Proposed } \\
\text { Compound }\end{array}$ & Enrichment \\
\hline 132.0303 & $\mathrm{C}_{4} \mathrm{H}_{6} \mathrm{NO}_{4}$ & 132.0302 & -0.0004 & 0.8 & Aspartic acid & Neuron \\
\hline 174.0405 & $\mathrm{C}_{6} \mathrm{H}_{8} \mathrm{NO}_{5}$ & 174.0402 & -0.0008 & 1.7 & $\mathrm{~N}$-acetylaspartate & Neuron \\
\hline 281.2478 & $\mathrm{C}_{18} \mathrm{H}_{33} \mathrm{O}_{2}$ & 281.2481 & 0.0003 & 1.1 & Oleic Acid & Neuron \\
\hline 302.0649 & $\mathrm{C}_{10} \mathrm{H}_{13} \mathrm{~N}_{5} \mathrm{O}_{4} \mathrm{Cl}$ & 302.0656 & 0.0007 & 2.3 & adenosine & Astrocyte \\
\hline 564.5336 & $\mathrm{C}_{36} \mathrm{H}_{70} \mathrm{NO}_{3}$ & 564.5361 & -0.0043 & 4.4 & Cer(d36:1) & Astrocyte \\
\hline 571.2877 & $\mathrm{C}_{25} \mathrm{H}_{48} \mathrm{O}_{12} \mathrm{P}$ & 571.2889 & -0.0012 & 2.1 & $\mathrm{PI}(16: 0)$ & Astrocyte \\
\hline 673.4785 & $\mathrm{C}_{37} \mathrm{H}_{70} \mathrm{O}_{8} \mathrm{P}$ & 673.4808 & 0.0049 & 3.4 & $\operatorname{PA}(34: 1)$ & Neuron \\
\hline 674.4789 & $\mathrm{C}_{36} \mathrm{H}_{69} \mathrm{NO}_{8} \mathrm{P}$ & 674.4761 & -0.0028 & 4.1 & $\operatorname{PE}(31: 1)$ & Neuron \\
\hline 726.5415 & $\mathrm{C}_{41} \mathrm{H}_{77} \mathrm{NO}_{7} \mathrm{P}$ & 726.5438 & 0.0057 & 3.2 & $\mathrm{PE}(36: 2)$ & Neuron \\
\hline 727.5251 & $\mathrm{C}_{41} \mathrm{H}_{76} \mathrm{O}_{8} \mathrm{P}$ & 727.5278 & 0.0057 & 3.7 & $\mathrm{PA}(38: 2)$ & Neuron \\
\hline 750.5421 & $\mathrm{C}_{43} \mathrm{H}_{77} \mathrm{NO}_{7} \mathrm{P}$ & 750.5438 & 0.0056 & 2.3 & $\operatorname{PE}(38: 4)$ & Neuron \\
\hline 773.5307 & $\mathrm{C}_{42} \mathrm{H}_{78} \mathrm{O}_{10} \mathrm{P}$ & 773.5333 & 0.0063 & 3.4 & $P G(36: 2)$ & Neuron \\
\hline 776.5577 & $\mathrm{C}_{45} \mathrm{H}_{79} \mathrm{NO}_{7} \mathrm{P}$ & 776.5600 & -0.0038 & 3.0 & $P E(P 40: 5)$ & Neuron \\
\hline 786.5262 & $\mathrm{C}_{42} \mathrm{H}_{77} \mathrm{NO}_{10} \mathrm{P}$ & 786.5285 & 0.0059 & 3.0 & PS(36:2) & Neuron \\
\hline 788.5419 & $\mathrm{C}_{42} \mathrm{H}_{79} \mathrm{NO}_{10} \mathrm{P}$ & 788.5442 & 0.0059 & 3.0 & $P S(36: 1)$ & Neuron \\
\hline 789.5408 & $\mathrm{C}_{46} \mathrm{H}_{78} \mathrm{O}_{8} \mathrm{P}$ & 789.5434 & 0.0018 & 3.3 & $\operatorname{PA}(43: 6)$ & Neuron \\
\hline 790.5364 & $\mathrm{C}_{45} \mathrm{H}_{77} \mathrm{NO}_{8} \mathrm{P}$ & 790.5387 & 0.0059 & 2.9 & $\operatorname{PE}(40: 6)$ & Neuron \\
\hline 797.5310 & $\mathrm{C}_{44} \mathrm{H}_{78} \mathrm{O}_{10} \mathrm{P}$ & 797.5333 & 0.0059 & 2.9 & $P G(38: 4)$ & Neuron \\
\hline 836.5248 & $\mathrm{C}_{43} \mathrm{H}_{80} \mathrm{NO}_{10} \mathrm{PCl}$ & 836.5208 & -0.007 & 4.7 & PS(37:2) & Neuron \\
\hline 837.5275 & $\mathrm{C}_{41} \mathrm{H}_{78} \mathrm{~N}_{2} \mathrm{O}_{13} \mathrm{P}$ & 837.5247 & -0.007 & -3.3 & $\begin{array}{l}\text { PE-GlcNAc- } \\
\text { DG(28:0) }\end{array}$ & Neuron \\
\hline 885.5469 & $\mathrm{C}_{47} \mathrm{H}_{82} \mathrm{O}_{13} \mathrm{P}$ & 885.5493 & 0.0068 & 2.7 & $\mathrm{Pl}(38: 4)$ & Neuron \\
\hline 887.5456 & $\mathrm{C}_{44} \mathrm{H}_{85} \mathrm{O}_{13} \mathrm{PCl}$ & 887.5422 & -0.0063 & 3.8 & $\mathrm{PI}(35: 0)$ & Neuron \\
\hline 888.6208 & $\mathrm{C}_{48} \mathrm{H}_{90} \mathrm{NO}_{11} \mathrm{~S}$ & 888.6235 & 0.007 & 3.0 & Sulfatide (24:1) & Astrocyte \\
\hline
\end{tabular}

